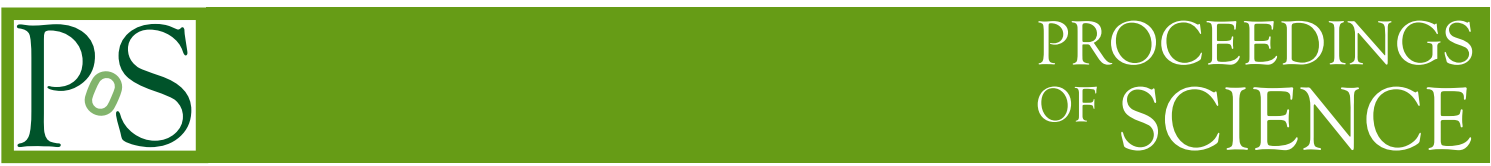

\title{
Variability of the GeV sky
}

\author{
Andrii Neronov ${ }^{a}$, Denys Malyshev ${ }^{b}$, Maria Chernyakova ${ }^{* d}$,Alexander Lutovinov ${ }^{e}$ \\ ${ }^{a}$ ISDC Data Center for Astrophysics, Chemin d'Ecogia 16, 1290 Versoix,Switzerland \\ ${ }^{b}$ Bogolyubov Institute for Theoretical Physics, 14-b Metrolohichna street, Kiev, 03680, Ukraine \\ ${ }^{c}$ Dublin City University, Glasnevin, Dublin 9, Ireland \\ ${ }^{b}$ DIAS, Fitzwiliam Place 31, Dublin 2, Ireland \\ d Space Research Institute (IKI), 84/32 Profsoyuznaya Str, Moscow 117997, Russia \\ E-mail: masha.chernyakova@dcu.ie
}

\begin{abstract}
High-energy gamma-ray emission from the Galactic plane above $\sim 100 \mathrm{MeV}$ is composed of three main contributions: diffuse emission from cosmic ray interactions in the interstellar medium, emission from extended sources, such as supernova remnants and pulsar wind nebulae, and emission from isolated compact source populations. The diffuse emission and emission from the extended sources provide the dominant contribution to the flux almost everywhere in the inner Galaxy, preventing the detection of isolated compact sources. In spite of this difficulty, compact sources in the Galactic plane can be singled out based on the variability properties of their $\gamma$-ray emission. Our aim is to find sources in the Fermi data that show long-term variability. We performed a systematic study of the emission variability from the Galactic plane, by constructing the variability maps. We find that emission from several directions along the Galactic plane is significantly variable on a time scale of months. These directions include, in addition to known variable Galactic sources and background blazars, the Galactic ridge region at positive Galactic longitudes and several regions containing young pulsars. We argue that variability on the time scale of months may be common to pulsars, originating from the inner parts of pulsar wind nebulae, similarly to what is observed in the Crab pulsar.
\end{abstract}

An INTEGRAL view of the high-energy sky (the first 10 years) - 9th INTEGRAL Workshop and celebration of the 10th anniversary of the launch

15-19 October 2012

Bibliotheque Nationale de France, Paris, France

${ }^{*}$ Speaker. 


\section{Inroduction}

Most of the $\gamma$-ray emission from the Galactic plane in the energy band above $0.1 \mathrm{GeV}$ is produced by cosmic ray interactions with interstellar matter (e.g. Abdo et al. (2009)). These interactions, on a time scale of $\sim 10^{7} \mathrm{yr}$ and on distance scales of hundreds of parsecs, result in the production of bright large-scale diffuse emission from the entire Galaxy. Isolated Galactic $\gamma$-ray sources, such as pulsars, pulsar wind nebulae, supernova remnants, and $\gamma$-ray-loud binary systems are superimposed on this large-scale diffuse emission. The strong diffuse background emission provides the main obstacle for detecting individual isolated sources in the Galactic plane. Inhomogeneities of the diffuse emission induced by the inhomogeneity of matter distribution in the interstellar medium lead to local brightness enhancements of the $\gamma$-ray emission from the Galactic plane, which might be misinterpreted as isolated sources. The most straightforward distinguishing property of isolated $\gamma$-ray sources is variability.

The only firmly established class of generically variable Galactic sources is that of $\gamma$-rayloud binaries. These systems, however, provide a very moderate contribution to the overall $\gamma$-ray emission from the Galaxy. Other known types of Galactic $\gamma$-ray sources, including the diffuse emission and supernova remnants are not variable on the day-to-month time scales. This feature could reveal the presence of $\gamma$-rayloud binaries even at the top of much stronger diffuse emission.

The recent discovery of $\gamma$-ray flaring activity of the Crab pulsar (Abdo et al. 2011) shows that pulsars (or the inner compact parts of the pulsar wind nebulae, PWNe) should also be considered as a possible variable Galactic source population. However, it is difficult to draw definitive conclusions about the variability of the entire pulsar population based on the study of a single source, especially taking into account the fact that the Crab pulsar could be considered as a special case because it is the youngest known $\gamma$-ray-loud pulsar. Our study shows, however, that long (month) time scale variability may indeed be a generic feature of pulsars/PWN systems (Neronov et al. 2012).

Fermi/LAT is a pair-conversion gamma-ray detector operating between $20 \mathrm{MeV}$ and $300 \mathrm{GeV}$. The LAT has a wide field of view of sr at $1 \mathrm{GeV}$, and observes the entire sky every two orbits. Therefore it is a perfect telescope for the systematical study of the variability of the GeV sky on a scale of hours-to-days.

\section{Variability Maps}

A simple way to verify if $\gamma$-ray emission from a given direction on the sky is variable or constant is to analyze the lightcurve and check if it is consistent with a constant flux. Several measures of (in)consistency with the constant flux can be considered.

Consider the flux $F\left(l, b, t_{i}\right)=F_{i}(l, b)$ in the given sky direction $(l, b)$ (in Galactic coordinates) binned in $N$ time bins $1 \leq i \leq N$. The flux is consistent with constant if the reduced $\chi^{2}$ of the fit of the lightcurve with the constant

$$
\chi^{2}(l, b)=\frac{1}{N-1} \sum_{i=1}^{N} \frac{\left(F_{i}-\bar{F}(l, b)\right)^{2}}{\sigma_{i}^{2}}
$$

is close to one (here $\sigma_{i}$ are the measurement errors in each time bin and $\bar{F}(l, b)$ is the average flux in the $(l, b)$ direction). If we consider the dependence of $\chi^{2}$ on $(l, b)$ we expect to find $\chi^{2} \simeq 1$ in 
all directions except for the directions in which there are variable isolated $\gamma$-ray sources. All nonvariable $\gamma$-ray sources, such as supernova remnants and the large-scale diffuse Galactic emission should not appear in the $\chi^{2}(l, b)$ map. Instead, the sources that are characterized by a variability amplitude larger than $\sim \sigma_{i} / \bar{F}(l, b)$ should be visible as excess in the $\chi^{2}$ map.

The scatter of the source flux around its average value, characterized by the $\chi^{2}$ of the fit of the lightcurve with a constant, provides a good measure of variability especially for sources that are systematically variable throughout the observation period. On the other hand, sources that produce on average one flare with a duration much shorter than the span of the observation period are gradually "washed out" from the $\chi^{2}(l, b)$ map as the overall exposure time increases. Indeed, the contribution of the time bins containing the flare to the overall $\chi^{2}$ given by Eq. (2.1) decreases with increasing number of bins. Accordingly, single-flare sources might disappear from the $\chi^{2}$ map with large exposure. A better measure of variability for the single-flare sources is the maximum deviation of the flux from the average value

$$
\sigma_{\max }(l, b)=\max _{i=1}^{N}\left(\sqrt{\frac{\left(F_{i}-\bar{F}(l, b)\right)^{2}}{\sigma_{i}^{2}}}\right) .
$$

Similarly to the $\chi^{2}$ measure, excess of $\sigma_{\max }$ beyond the expected statistical fluctuations indicates the presence of a flaring source in a given sky direction. In contrast to the $\chi^{2}$ measure, the $\sigma_{\max }$ measure does not decrease with increasing exposure.

A systematic view on the entire population of variable sources in the Galaxy (both systematically variable and flaring) can be obtained by identifying all significant excesses in the $\chi^{2}(l, b)$ and $\sigma_{\text {max }}(l, b)$ maps constructed based on the $\gamma$-ray lightcurves in different energy bands and different energy scales.

In our analysis we used all publicly available LAT data for the period from August, 2008 to October, 2011.

\section{Variability of emission from the inner part of the Galactic disk}

Fig. 1 shows sky regions containing the known variable Galactic $\gamma$-ray sources LSI +61303 , Cyg X-3 and V407 Cyg. All three sources are $\gamma$-ray-loud binaries with quite different types of variability. LSI +61303 is a persistent source, variable on $26.4 \mathrm{~d}$ orbital period and on a longer superorbital period of $4.6 \mathrm{yr}$. Cyg X-3 is a recurrent flaring source that already exhibited several bright flares over the $3.5 \mathrm{yr}$ period of Fermi/LAT exposure. V407 Cyg is a symbiotic binary that produced only one flare during the LAT exposure. One can see that in spite of the large difference in the variability properties of the sources, all three sources are clearly detected as strong excesses in the variability map, shown by the green contours. The other excesses above and below the Galactic plane are produced by known blazars.

Fig. 2 shows the region around the Galactic Center. The excess variability is not centered at the Galactic Center itself (the bright source in the center of the image). Instead it traces the part of the Galactic plane at positive Galactic longitude $0^{\circ}<l<7^{\circ}$ dominated by diffuse emission, with no clearly isolated bright sources. The end part of the variable region, with the brightest excess at the level of $\sigma_{\max } \simeq 4.5$, is centered at the bright isolated source identified with supernova remnant 

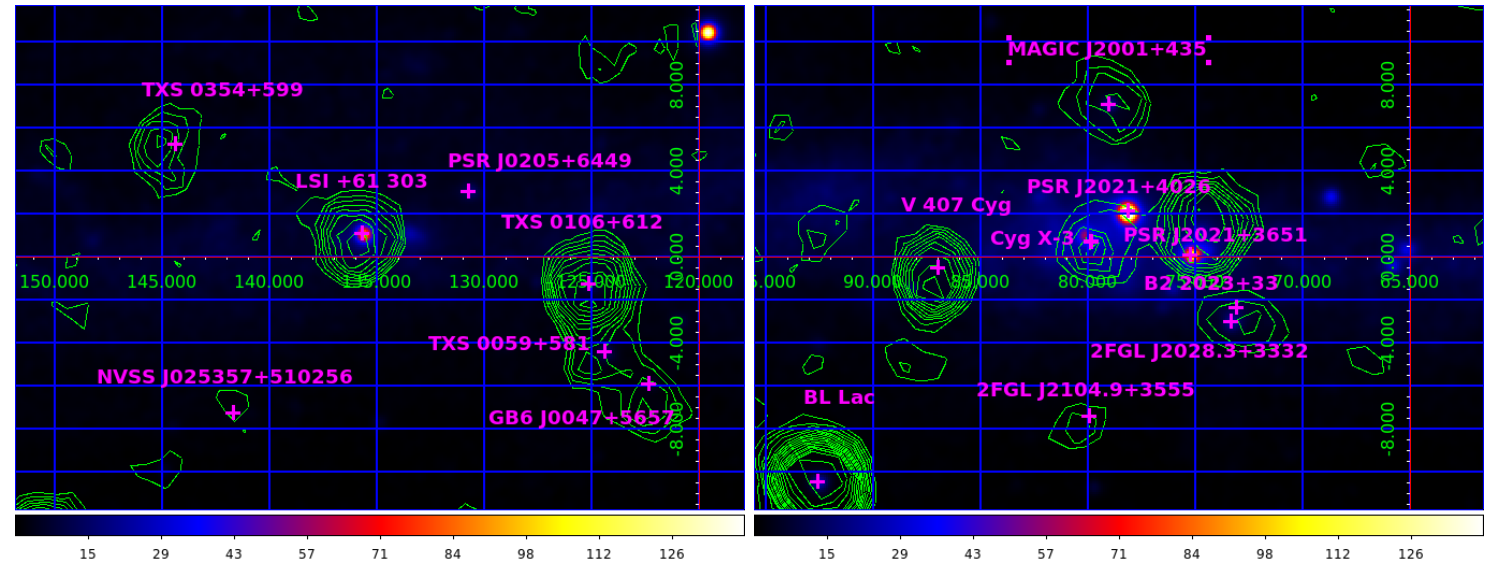

Figure 1: LAT count map in the energy range above $300 \mathrm{MeV}$ with the superimposed contours showing the $\sigma_{\max }$ values found in the lightcurves binned into the month-long time bins. Contour levels start from $3 \sigma$ with steps of $0.5 \sigma$.

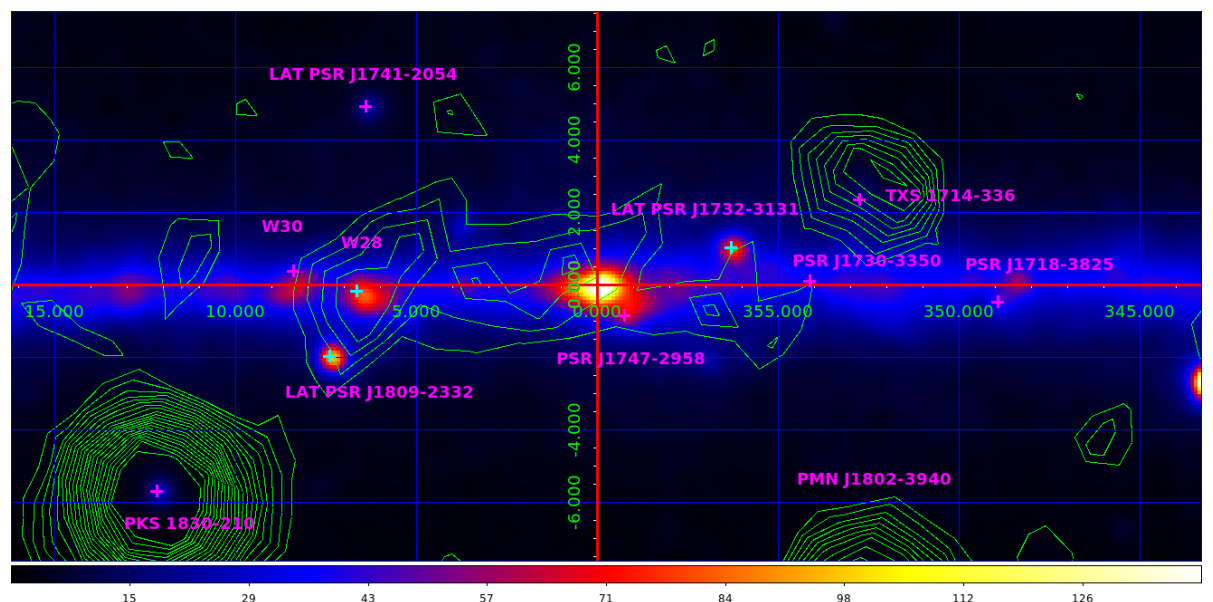

Figure 2: LAT count map with superimposed variability contours for the region around the Galactic Center. Contour levels start from $3 \sigma$ with steps of $0.5 \sigma$.

W28. Appearance of excess variability from the region of diffuse emission and from a supernova remnant is surprising.

Another region with excess variability is found in the direction of the Kookaburra pulsar wind nebula complex, shown in Fig. 3. This sky region contains three young pulsars, PSR J1418-6068, PSR J1410-6132 and PSR J1413-6205, which emit pulsed $\gamma$-ray emission in the 0.1-10 GeV band. Similarly to the regions of the Galactic ridge and of LSI +61 303, Cyg X-3 / V407 Cyg, the variable sources above and below the Galactic plane, visible on the map, are background blazars. The source to the right of the Kookaburra complex, PSR B1259-63, is not visible in the three-year count map (shown by the color scale), but appears as a very bright excess in the variability map. This is not surprising, because this source is a $\gamma$-ray-loud binary with a radio pulsar on a highly eccentric orbit with a $3.5 \mathrm{yr}$ orbital period. It exhibits periods of activity only close to the periastron passage. The last periastron passage happened in December 2010. The source produced a bright one-month-long 


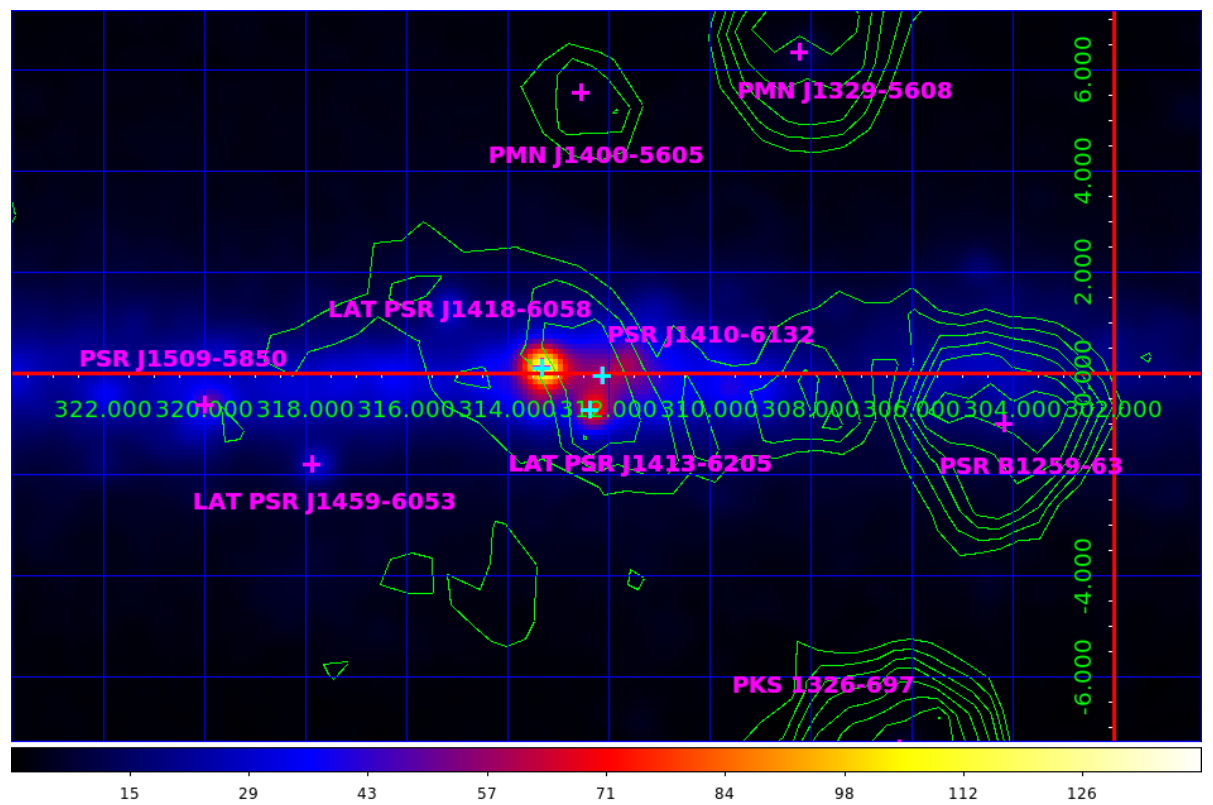

Figure 3: LAT count map and variability contours in the direction of sky region containing pulsars PSR J1418-6068, PSR J1410-6132 and PSR J1413-6205.

flare in January 2011 and disappeared since then.

Excess of variability on a scale of months at the level $\sigma_{\max } \simeq 15$ is also detected from the direction of the Crab pulsar.

\section{Discussion}

Emission variability from the Galactic plane is caused by compact $\gamma$-ray sources. The only firmly established class of generically variable Galactic sources is that of $\gamma$-ray-loud binaries. In this respect, it is not surprising that the highest excess variability is found in the directions of known $\gamma$-ray-loud binary systems: LSI +61 303, V407 Cyg, Cyg X-3, and PSR B1259-63.

Additionally, significant variability is found in the direction of the region of the Kookaburra nebula, which contains several young pulsars. Unfortunately, the point spread function of LAT in the energy band in which the variability is detected is too large to tell if one of the pulsars produces variable emission on a scale of months, or if the variability is the result of the variability of several pulsars. It is also not clear why pulsars in the Kookaburra region would be variable while other young $\gamma$-ray-loud pulsars in the Galactic plane do not reveal any excess variability a time scale of months. Additional study is needed to clarify the origin of $\gamma$-ray emission from young pulsars, including the youngest Crab pulsar.

Another region that exhibits excess variability is the Galactic ridge at positive Galactic longitudes $0^{\circ}<l<7^{\circ}$. The strongest excess of variability is centered at the supernova remnant W28. Clearly, the variability in this sky direction cannot be produced by the extended emission from parts of W28 itself, which have extension of about $0.5^{\circ}$, i.e. $\sim 20 \mathrm{pc}$ at $\sim 2 \mathrm{kpc}$. The variable region contains several young pulsars, including PSR J1747-2809 and PSR J1746-2850, close to 
the Galactic Center with the ages $T=5.3 \mathrm{kyr}$ and $T=12.7 \mathrm{kyr}$, just slightly older than those of the Crab pulsar. Closer to the W28 remnant there is PSR B1757-24, a pulsar of age $T=15.5 \mathrm{kyr}$. Finally, W28 itself is supposed to be associated with the pulsar PSR 1758-23. It is possible that the variability is produced by one or several pulsars in this region of the Galaxy. Otherwise, the source of variability could be $\gamma$-ray-loud binaries that are below the sensitivity of LAT.

\section{Conclusions}

To summarize, we have studied variability of $\gamma$-ray emission on a time scale of months from the Galactic plane in the energy band above $300 \mathrm{MeV}$ in the Fermi/LAT data and found several directions with significantly variable emission. We found that strong variability excess is detected in the directions of known $\gamma$-ray-loud binary systems. Remarkably, there are no signatures of weaker variability, which could be clearly associated to other binary systems with black holes and/or neutron stars. At the same time, our study revealed weaker variability excess in the directions of pulsars. This indicates that long variability on a scale of months, as recently discovered in the Crab pulsar, may be a generic feature of the $\gamma$-ray emission from the young pulsar population, so that pulsar/PWN systems should be considered as variable Galactic $\gamma$-ray sources. We also remark that the position of this variable region is consistent with the positions of sources that are variable in hard X-ray band (see e.g. Telezhinsky et al. (2010)).

The threshold value $\sigma_{t h r}$ for significant detection of variability is different for the search of variable emission from an arbitrary direction along the Galactic plane and for the search of variability of emission from known isolated $\gamma$-ray source classes. If we are interested in the variability properties of known sources, the threshold $\sigma_{t h r}$ can be decreased, because in this case there is no trial factor associated to the scan of the entire Galactic plane. The probability to find an excess variability at the level above $3.5 \sigma$ is just about $1 \%$ for each individual source. Excess variability at a level higher than $3.5 \sigma$ is found in two more directions on the sky, which coincide with the positions of the known sources, PSR J1826-1256 and PSR J1119-6127.

Evidence for excess variability at the locations of these pulsars, if combined with the detection of variability from the direction of Kookaburra region that contains several young pulsars, shows that long time scale variability may be a generic property of young pulsars, and not a peculiar feature of the Crab pulsar alone.

\section{References}

Abdo, A. A., Ackermann, M., Ajello, M., et al. 2011, Science, 331, 739

Abdo, A. A., Ackermann, M., Ajello, M., et al. 2009, Physical Review Letters, 103, 251101

Neronov, A., Malyshev, D., Chernyakova, M., \& Lutovinov, A. 2012, A\&A, 543, L9

Telezhinsky, I., Eckert, D., Savchenko, V., et al. 2010, A\&A, 522, A68 